\title{
Et Dieu créa Aïcha Qondicha :
}

femmes sacrées et femmes maléfiques dans le maraboutisme tunisien

\section{Salem AKRIMI}

\section{OpenEdition}

\section{Journals}

Édition électronique

URL : https://journals.openedition.org/leportique/899

DOI : 10.4000/leportique.899

ISSN : $1777-5280$

Éditeur

Association "Les Amis du Portique"

Référence électronique

Salem AKRIMI, «Et Dieu créa Aïcha Qondicha : », Le Portique [En ligne], e-Portique, mis en ligne le 26 décembre 2006, consulté le 10 septembre 2022. URL : http://journals.openedition.org/leportique/899 ; DOI : https://doi.org/10.4000/leportique.899

Ce document a été généré automatiquement le 10 septembre 2022

Tous droits réservés 


\title{
Et Dieu créa Aïcha Qondicha :
}

\author{
femmes sacrées et femmes maléfiques dans le maraboutisme tunisien
}

\section{Salem AKRIMI}

1 Du Maghreb aux régions de l'Asie où l'islam exerce une influence, de nombreux personnages ont été désignés comme doués de baraka. Selon ces différentes populations, la baraka est considérée comme une prérogative de Dieu possédée par les prophètes et les saints. Cet afflux leur permet de réaliser des miracles comme les guérisons, la divination, l'exorcisme, les intercessions etc.. de nos jours ces personnages existent toujours. En Afrique du Nord - aire culturelle à laquelle cette étude est confrontée - il est devenu courant de les qualifier par le terme de «marabout ", même si peuvent être rangés sous ce qualificatif des personnages aussi divers que le sorcier du village, le taleb - théologien ayant acquis par l'étude des techniques de guérison - ou encore le soufi - personnage versé dans l'ésotérisme islamique ${ }^{1}$.

Durant la consultation de nombreux ouvrages portant sur cette notion de baraka, nous avons remarqué que rares étaient les études consacrées aux femmes détentrices de cette vertu ${ }^{2}$. Or, à Bir El Haffey, petite ville du centre tunisien ${ }^{3}$ où nous avons mené nos investigations, de nombreuses femmes sont jugées comme détentrices de baraka. Ces représentantes féminines du maraboutisme sont désignées par les termes indigènes de 'arrāf $a^{4}$ ou de mabrū $k a^{5}$. Les personnes qui qualifient ces femmes voguent entre ces deux termes en fonction du prestige qu'elles leur attribuent, le terme de mabrūka étant beaucoup plus honorifique. Au seul regard de ces qualificatifs, les praticiennes de la sphère magico-religieuse auraient donc été admises dans la sphère publique qui, selon les monographies de cette région, est traditionnellement réservée aux hommes. Pour en témoigner, Jean Duvignaud précise dans sa magnifique étude intitulée Chébika, Changements dans un village du Sud Tunisien, que «le rôle de la femme [tunisienne] est devenu, malgré le mépris qui l'affecte apparemment, infiniment plus important que celui de l'homme dont elles contrôlent en groupe les réactions: la sexualité, l'attachement des enfants et la nourriture. Maîtresse de la reproduction et de la manducation, la femme islamique est devenue dès l'ancienne Tunisie, un élément caché mais actif, jamais avoué, de la vie publique. $»^{6}$ Or, tout don résulte d'une sanction de la 
communauté. Sur notre terrain, un proverbe stipule : «il n'y a pas de prophète sans miracle et il n'y a pas de saints qui n'exercent de choses extraordinaires (la nabiyon illā bi al-mo'djizāti wa la waliyon illā bi al-karāmāti)». Si les femmes de cette région doivent plutôt constituer un élément discret de leur environnement, comment peuvent-elles à ce moment témoigner de baraka?

3 Avant de répondre à cette question, nous présenterons l'état des pratiques magicoreligieuses féminines à Bir El Haffey, plus particulièrement le parcours d'une de ses principales actrices. Nous tenterons ensuite de voir s'il est possible de rapprocher cette sphère de la société tunisienne aux autres grands courants maghrébins étudiés par les ethnologues. Enfin, nous répondrons au dilemme posé par l'élection en analysant la manière dont ces femmes s'y sont prises pour imposer leurs personnalités en dépit du regard malveillant de certains hommes.

La mosquée des femmes de Bir El Haffey

4 Dans son ouvrage intitulé Possession, magie et prophétie en Algérie, Aïssa Ouitis observait que «dans les Bibans les femmes ne participaient à aucune manifestation religieuse publique. On ne voit pas comment elles auraient pu faire autrement dans la mesure où les mosquées n'étaient composées que d'une salle unique (au lieu de comprendre une double compartimentation comme on en trouve actuellement) et que la promiscuité des sexes était proscrite. Cette architecture revenait donc à interdire pratiquement l'accès des femmes aux mosquées. Le groupe féminin s'est alors rabattu dans sa pratique quotidienne, sur un ensemble de cultes beaucoup plus "païens" qu'islamiques : arbres réputés marabout, génies des lieux, et bien entendu sur le culte des saints: ainsi, si les jours de fêtes religieuses sont, pour les hommes, autant d'occasion d'organiser des prières collectives, ils sont, pour les femmes, celles de faire la tournée des saints du village et des dechra voisines. Bien sûr, l'observation des règles de l'Islam n'est pas absente dans le culte féminin, mais elle n'intervient que bien plus tard, vers la vieillesse. $»^{7}$ À Bir El Haffey, les mosquées se trouvent au centre de la commune, le souk, endroit proscrit aux femmes qui se respectent. Cependant ces femmes font également oeuvre de piété : elles ont pour habitude de visiter une bâtisse située aux pieds de la montagne, considérée comme imprégnée de baraka. Cette bâtisse est le tombeau de Jédi Ali qui, d'après la légende, serait un descendant du gendre et neveu du prophète de l'Islam, Ali ben Abi Taleb. C'est ce que rapporte une pièce de marbre dressée sur sa tombe. Toujours d'après la légende, Jédi Ali serait originaire de la place mythique de la Sagia El Hamra, région marocaine d'où sont originaires tous les saints du Maghreb et qui aurait été foulée par le sultan des saints, Sidi Abd El Kader Djilani, figure connue de toute l'aire arabo-musulmane.

5 Le tombeau de Jédi Ali fait l'objet de nombreuses visites. Nous avons remarqué que ce sont surtout les femmes qui s'y rendent. Les drapeaux sont déployés sur le marabout ${ }^{8}$. De nombreuses personnes viennent se recueillir sur la tombe et faire don de quelques dinars dans l'espoir de profiter de l'aura du lieu. Parfois même elles sacrifient des bêtes, mangent du couscous et rencontrent la gardienne du tombeau, Jamila.

6 Nous avons pu discuter avec la mère d'un jeune garçon qui décida de consulter Jamila. Oussama, son fils, était régulièrement atteint de fièvre et frappé par des diarrhées. La cause de ses maux était, selon la famille, peut-être due au mauvais œil. Ce trouble, connu à Bir El Haffey sous les termes de 'ayn ou nafs, provient d'une attaque magique du regard et a pour conséquence d'atteindre l'âme de la victime. Il s'agit d'un trouble 
de l'identité que tout thérapeute traditionnel doit être capable de prendre en charge et ce, dès le début de son initiation aux soins magico-religieux.

7 La maman d'Oussama, Om El Khir', ne se résigna pas et décida de rencontrer Jamila. Avant de se rendre au tombeau de Jédi Ali, elle avait pris soin de prendre des herbes destinées à enfumer le lieu saint. Sur les lieux, Jamila était assise au centre d'un groupe de femmes de tous âges. Devant Jamila se trouvaient divers objets destinés à la relation entre le pèlerin, le saint et la baraka, entre autres les pierres que l'on jette dans le brasero (kānūn), des bougies, des herbes et une substance connue sous l'appellation arabe de kamūn. Le kamūn est une graine noire ou verte qui sert à aromatiser les repas mais qui est également utilisée comme médicament pour chasser les mauvais génies ${ }^{10}$. Pour répondre aux sollicitations d'Om El Khir, Jamila mélangea avec sa salive ces graines de kamūn et les apposa sur le front d'Oussama. Elle les apposa avec sa main gauche, la seule main autorisée pour effectuer ce geste.

De la possession...

8 Jamila Akrimi est née à Bir El Haffey en 1956. Elle est mariée et mère de quatre enfants. Toute la communauté de Bir El Haffey attribue à Jamila un caractère sacré. Les gens la décrivirent comme digne héritière de Jédi Ali. Cependant, ce caractère sacré, cette baraka, n'émane pas d'elle-même. Jamila nous dira qu'elle n'est qu'un canal par lequel Dieu décide d'apporter de l'aide à autrui. Jamila ne se consacre qu'aux autres avec pour seule technique l'apposition de sa main gauche. Chaque personne malade qui rentre chez elle est censée se sentir mieux dès son départ.

9 Parfois, Jamila est comme absente. Elle semble communiquer avec des êtres immatériels, venus de pays lointains. Elle voyage. Elle visite d'autres contrées, tout comme dans les récits hagiographiques de grands saints et tout comme son ancêtre Jédi Ali, Jamila a visité la Sagia El Hamra. Lorsqu'elle était en pleine méditation, elle a senti qu'elle voyageait ; "son corps était ici mais elle était ailleurs ». Grâce à son don, elle a visité tous les pays, même La Mecque, comme pèlerine.

Jamila _ La baraka c'est le fruit d'un grand travail, d'un travail acharné... de fatigue, d'épuisement, de... patience. La baraka, c'est aussi une souffrance physique. Quelquefois mon cœur bat très fort. J'ai les pieds lourds. Je ne peux pas marcher. Tout ça ce sont les conséquences de cette baraka. Des fois je me prive de manger... pendant plusieurs jours. La première fois, je suis même restée un mois sans manger. Sans parler. Je ne communiquais qu'avec des gestes. Les saints ont voulu ça. C'est malgré moi que je souffre. Je n'ai pas la sensation d'avoir faim, ni celle d'avoir soif. Je ne sens rien.

Dès le début de l'adolescence, vers l'âge de douze ans - probablement à sa puberté Jamila voit des choses étranges. Elle a des hallucinations. Elle voit des serpents, des lions, des dromadaires. Ces signes sont généralement interprétés comme les premières manifestations de la baraka :

Jamila _ Quelquefois je m'évanouis, quelquefois j'ai peur, je vois des choses, ce sont des choses monstrueuses, qui font mal.

Ethnologue [ethn]_ Des maux physiques? Des maux psychologiques?

Jamila_Oui! Les deux!

Ethn _ Les deux. Et pendant combien de temps vous avez vécu ça?

Jamila _ Alors selon les cas... quelquefois la durée est longue, quelquefois elle est légère, quelques heures, quelquefois un jour, quelquefois dix jours. Selon le cas.

Ethn_Pendant combien d'années? Maintenant vous ressentez encore ces choses? Jamila_ Non! Non! 
Ethn _ Non ? c'est parti ? Depuis combien de temps?

Jamila _ Depuis quatre ans.

Ethn _ Depuis quatre ans?

Jamila _ Depuis quatre ans, j'ai compris que je n'ai pas ce mal. J'ai compris que je ne suis pas malade.

Ethn _ Et la première chose ? Vous vous souvenez de la première chose que vous avez

eue à onze ans ? C'était quoi ? La première sensation bizarre?

Jamila _ Euh!

Ethn _ La première chose anormale que vous ayez ressentie?

Jamila _ Quand j'étais petite?

Ethn_Oui!

Jamila _ Alors j'ai vu un grand serpent.

Ethn _ Un grand serpent?

Jamila _ J'ai vu un grand serpent.

Ethn__'accord!

Jamila _ J'ai vu un grand serpent qui m'a fait peur et qui est allé. Il s'en est allé sans me toucher, sans me...

Ethn _ Un vrai « grand serpent »? pas une hallucination, un vrai « grand serpent »?

Jamila _ Je sais pas. C'était peut-être un esprit.

11 C'est à ce moment que Jamila commence à montrer des signes de faiblesse. Elle se prive de manger, de boire, refuse toute relation sociale. À l'époque de notre recherche ${ }^{11}$, cela faisait cinq années qu'elle apportait de l'aide aux malades. Jamila aurait été chargée par son ancêtre Jédi Ali d'imposer sa main gauche sur les malades. Cette gestuelle peut être considérée comme plus que de l'altruisme ou de la sympathie pour ses consultants. Elle est à mettre au rang de l'empathie :

Jamila _ Dieu est généreux. Lorsque quelqu'un est malade et qu'il vient me visiter en consultation, même s'il ne parle pas, je sens ce qu'il a. Lorsqu'il a mal à la tête, je sens qu'il a mal à la tête. Lorsque cette personne-là a mal au ventre, je sens qu'elle a mal au ventre. Lorsqu'une personne a mal au dos, je sens qu'elle a mal au dos aussi.

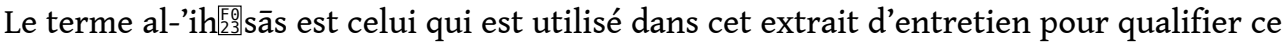
qu'elle ressent chez le patient. À chaque personne à qui nous avons demandé si elle

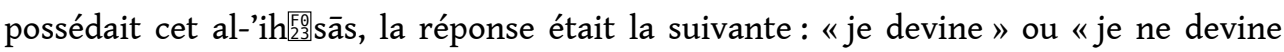
pas». Cependant, en contrepartie de ce don, Jamila eut affaire à une sensibilité exacerbée. Cette sensibilité, qui était au départ une peur, se transforma, selon elle, en amour. Elle réussit à retourner ses émotions et à en faire un moyen par lequel elle pouvait apporter de l'aide à autrui. Mais, avant de faire de cette sensibilité une force constructrice, Jamila eut à surmonter l'épreuve de la possession par les génies, djinn, ces êtres créés selon le Coran non pas comme les hommes à base d'argile mais à partir d'un feu subtil ou encore d'une fumée sans feu. Ces êtres constituent, avec les hommes, le diable et les anges, une des quatre créatures de Dieu doués d'intelligence. Cependant les anges, contrairement aux trois autres créatures, sont des êtres asexués ${ }^{12}$.

Dans la région de Bir El Haffey, les deux termes qui traduisent l'état de possession par les génies sont ceux d'al-libsu et d'al-'iltibās. Ces termes ont un sens très proche. Ils indiquent l'état où l'individu perd tout contrôle sur lui-même, toute maîtrise de ses

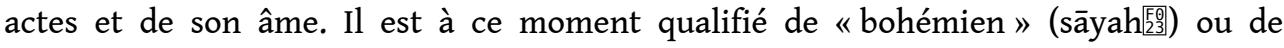
" charlatan » (musha'widh). Il est censé ne plus accorder d'importance à ses vêtements, à sa nourriture... Ce qui, sur notre terrain, constitue un signe d'élection et prouve le 
détachement de l'individu avec son environnement. Il est soupçonné d'être en relation avec d'autres forces.

Or, en approfondissant l'analyse de ces termes, apparaissent des distinctions de valeur : du point de vue langagier, al-'iltibās signifie « ce avec quoi on se vêtit ». Al-libsu c'est «la coopération, la réunion avec autrui ». En langue arabe, il existe également des variations de ces mots à partir de la même racine :

concernant le terme, al-'iltibās, sa première variante est al-talabbusu. Il s'agit de "tout ce que met la personne » ou " tout ce qui sera mis sur la personne ». La deuxième variation, al-talbīs, signifie « accuser une personne sans preuve ». concernant le terme d'al-libsu, une de ses variantes est le mot libās : ce sont " les couronnes des fleurs "; l'autre variante est al-lubsa, "le vêtement ». Lubs ou labūs signifie "ce qui enveloppe le corps : l'armure ou le gilet $"$.

Dans tous les cas de figures, tout se passe comme si le djinn représente le vêtement porté par la personne. Il s'agit de la possession.

18 Cependant le terme d'al-'iltibās renvoie à une conception plus passive de l'individu avec la nature qui le possède, alors qu'al-libsu semble signifier une coopération avec cette même nature. Une possession maîtrisée et plus nécessairement subite, renvoie à un degré supérieur d'initiation. Nous assistons là à la distinction qui a souvent lieu entre l'exorcisme et l'adorcisme : l'exorcisme étant une simple pratique d'expulsion de la nature qui possède l'individu alors que l'adorcisme représente une pratique qui vise à créer un pacte de coopération entre l'individu et la nature qui le possède.

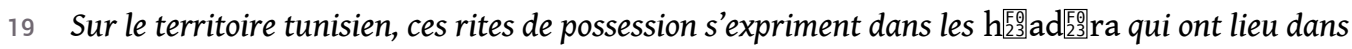

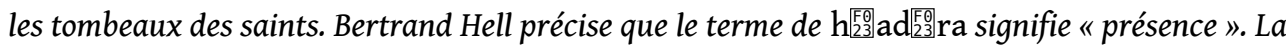

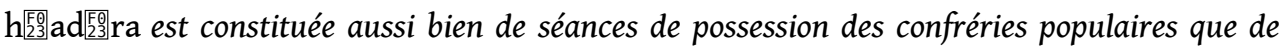
rites d'extase collectifs dans les marabouts ou des réunions spirituelles des ordres soufis ${ }^{13}$. Nous

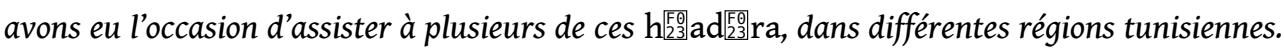
Généralement, les principales actrices de ces cérémonies sont les femmes. Avant les visites aux marabouts, ces dernières se trouvent souvent dans des états de fatigues physiques et

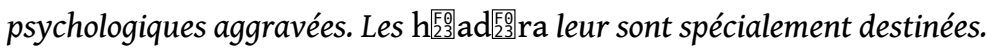

20 Lors de ces cérémonies, l'assistance est généralement importante. L'atmosphère est parfumée d'encens. À proximité de la tombe du saint, parfois de sa descendance - les dépouilles des enfants reposent auprès de celles des saints - se trouvent des percussionnistes. Leurs percussions sont toutes des bindīir ${ }^{14}$.

21 Les hommes tenant un bindir commencent à jouer et à chanter (ces chants sont des chants du Coran, des louanges au saint du tombeau, ainsi que des implorations pour la guérison des malades). Le centre de la piste située devant eux est destiné à recevoir les malades. Dès le premier tambourinement, les malades sont prises. Elles commencent à agiter le haut de leurs corps et à se balancer d'avant en arrière sur le même rythme que les percussions. Lorsque les joueurs de bindir arrêtent la musique et les chants, elles s'effondrent sur le sol. Quand ils reprennent, elles se lèvent et sautent pieds joints en faisant tourner leurs longues chevelures. Successivement ces femmes s'agitent, s'effondrent et effectuent des va-et-vient vers les dépouilles sacrées. Jamila, notre informatrice, vécut une expérience identique :

Jamila _ À la visite des marabouts, je sens quelque chose qui me possède. Dès fois, je sens quelque chose qui m'a soulevée, qui m'a embrassée, qui m'a tenue dans ses mains, soulevée. 
Ethn _ Est-ce que vous étiez prise comme les femmes que j'ai vues dans les tombeaux?

Est-ce que...vous aviez des mouvements avec votre corps, tout ça!

Jamila _ Je sens quelque chose. C'est le saint qui m'a accueillie. Je sens qu'il est à

côté de moi. Pas que Jédi Ali. À chaque marabout que je visite le saint est présent.

Ethn _ Tous ceux que vous visitez vous les sentez?

Jamila_Tous, tous!

Ethn _ Pendant cet état où vous êtes prise?

Jamila _ Je me sens chaude, j'ai de la sueur qui coule, je sens que quelqu'un est présent.

Ethn _ Vous êtes prise?

Jamila _ Oui.

L'amour entre son grand-père ou les autres saints et Jamila est envahissant et parfois ambigu. Jamila ressent la possession car elle est le récepteur de cet amour. La baraka est reproduite mythiquement et d'après les observations ethnologiques, au sein d'une même lignée. À la suite de cette fécondation, la force sauvage de l'individu, ses tourments, s'estompent et lui permettent de se mouvoir dans son environnement. La cure fut bénéfique pour Jamila lorsqu'elle devina l'identité de l'entité qui la possédait. Des thérapeutes traditionnels l'auraient aidée à prendre conscience de cette élection. Aujourd'hui, Jamila est également capable de diriger des cures.

Aïcha Qondicha versus Sidi Abd El Kader Djilani

Selon Bertrand Hell, lorsque, dans les cérémonies Gnawa marocaines, les possessions explosent de manière soudaine, provoquant angoisse dans l'assistance, c'est qu'un génie très particulier a fait irruption: Aïcha Qondicha. "L'atmosphère qui règne à présent tranche nettement avec celle des autres possessions. Certes, les sanglots et les trépignements sont identiques, mais l'heure n'est ni à l'exaltation mystique [...] ni à l'impétuosité [...] Non, il s'agit plutôt d'une sourde anxiété comme si se faisait sentir une menace confusément associée à la valence obscure, oppressante de la féminité profonde. Effectivement, dans la cour, la présence des femmes est devenue étouffante : sur l'aire de danse, un magma indistinct de poitrines ballottantes et de gorges râlantes ; dans les ténèbres autour de nous, partout des silhouettes qui s'agitent les cheveux dénoués et les bras tordus, en poussant des gémissements équivoques. Portée par les possédés, une inquiétante lame de fond semble en mesure de submerger la demeure. $»^{15}$ D'après Bertrand Hell (et de nombreux autres spécialistes ${ }^{16}$ ), l'apparition d'Aïcha Qondicha est toujours synonyme de malaise et d'inquiétude. Certes, l'identité de ce fantôme peut varier selon les contrées et Aïcha Qondicha n'est pas appelée de la même manière en Tunisie. D'ailleurs, pour Bertrand Hell, « Préciser la nature exacte d'Aïcha Qondicha paraît tâche impossible tant ses visages sont multiples. À son propos, les historiens avancent l'idée d'une antique déesse de la méditerranée orientale dont le culte serait diffusé en Afrique du Nord grâce aux phéniciens. La racine sémitique (QDS) de son nom renvoie à l'idée de sacré, la Qedecha des Cananéens présidait à la prostitution rituelle dans les temples. Au Maroc, à la plus grande colère des rigoristes, des prostituées ont encore droit de cité dans plusieurs sanctuaires [...]».

Malgré la pluralité de son identité partout où ce fantôme vient hanter les lieux la symbolique est la même: "les spécialistes de la littérature orale l'identifient soit comme l'archétype de "la mère castratrice", soit comme "l'anti-femme" par excellence, tentatrice d'un côté, oppressante et dévorante de l'autre. ${ }^{17}$ Les praticiennes de la sphère magico-religieuse tunisienne ne reproduiraient-elles pas la vie de cette femme somme toute séduisante? Aïcha Qondicha les doterait ainsi de sa trempe dans le but de 
pallier à la dépossession dont elles ont fait l'objet à l'origine. Aïcha Qondicha pourrait alors être assimilée au pendant féminin de Sidi Abd El Kader Djilani, le «sultan des saints » :

Au nom de Dieu écoute-moi si tu es habile / Des paroles mûrement réfléchies / Que j'apporte en son honneur / Son nom est le sultan des saints / Ses membres sont beaux, son visage éblouissant / Le père Moïse fils de la mère de la prospérité Les hommes, en raison de ma nostalgie, mon esprit s'envole / Je ne possède qu'un stylo et mon écriture est lourde / Il m'a attrapé avec un crochet et m'a ceinturé la taille / Il m'a piégée de la même manière qu'un oiseau / Il m'a tiré deux flèches dans le cœur / Il a transformé mes larmes en rivière

Toi le Créateur de l'horizon, Toi l'observateur / Mon cœur se consume telles les braises / Mes louanges vers lui sont mûres / Allège ce qui m'est pénible / Et donne au tendre Abd El Kader une missive

Il a monté la brune et chevauché avec elle / Et hélas mon Dieu, point de bruits de sabots / Sur ses traces un peuple [de dijinn] très important / Cache l'horizon, les monts et les plaines / Un bâton, un tamis, une tasse et un puits / Ses proches gouvernent au nom de la charia / Et l'égaré succombe dans le puits

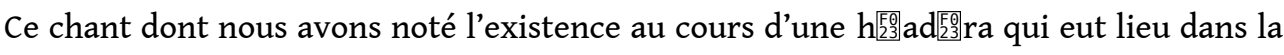
ville d'Om El Ksab, ville située dans le gouvernorat de Kasserine au centre ouest tunisien, est dédié au fameux Sidi Abd El Kader Djilani ${ }^{18}$. Nous avons vu dans l'introduction que s'en remettre à $\mathrm{Abd} \mathrm{El}$ Kader c'est nourrir le désir d'appartenir à une filiation douée de baraka. La plupart des personnes douées pensent descendre de cet homme légendaire, censé avoir accompli les plus nombreux et les plus spectaculaires des charismes. Sa mémoire est omniprésente chez les personnes pratiquantes du maraboutisme.

En analysant ce chant, nous observons que les qualités attribuées aux figures ancestrales sont celles de la force, de la puissance et de la prospérité. Pour donner du sens à ces qualités, le saint est toujours censé commander aux djinn : en se référant au Tourbillon des génies de Bertrand Hell, nous apprenons que Abd El Kader était un exorciste hors-pair, l'exorcisme représentant une des fins premières du maraboutisme, mais aussi une des étapes vers un rapport initiatique entre le thérapeute et la personne souffrante. L'auteur nous relate une cérémonie ayant eu lieu dans la banlieue de Marrakech, cérémonie qui fut le théâtre de la possession. La texture des chants recueillis par Bertrand Hell est semblable à celle que nous rapportons et ne diffère pas de nos analyses sur la possession. Dans la cérémonie qu'il décrit, au moment où est invoqué Abd El Kader, la figure paternelle, les corps se relâchent, alors que souvent, devant le père, les enfants doivent paraître dignes. Mais le maraboutisme est le lieu où l'on peut tout se permettre. Ici, ce sont les contacts avec les djinn qui mettent les corps en ébullition. Bien sûr sous le regard bienveillant du père Abd El Kader : "ô Djîlanî donne-nous la force, ô guéris-nous! $»^{19}$. La fureur des djinn doit être maîtrisée : «ô je suis entre tes mains ; ô regarde moi $\aleph^{20}$. Certes, la cérémonie décrite par Bertrand Hell procède bien différemment de celles que nous avons observées, mais l'urgence est la même.

Pour pallier cette urgence, moment où le seul capital dont on dispose s'échappe, le corps, la parole doivent être pris en charge par un initié. Dans le chant introduit auparavant, ce qui nous intéresse plus particulièrement, ce sont les qualités (ou défauts) qui sont censées échoir à la personne souffrante (présentes dans les deuxième et troisième couplets) et non pas à $\mathrm{Abd} \mathrm{El}$ Kader (ces dernières étant difficilement discutables, situons-nous à la veille de leur épanouissement). Que cette personne 
souffrante soit une femme ou un homme, ces qualités sont tout l'inverse : bon œil ( « ô je suis entre tes mains ») contre mauvais œil (" ô regarde moi, ô guéris moi »). Or, avant de pénétrer dans la sphère maraboutique, qu'il s'agisse d'une demande de guérison ou d'une future initiation, les individus se trouvent toujours, au départ, dans des situations pénibles à vivre. Les mots qui reviennent la plupart du temps sont ceux de " nostalgie ", " peine", " douleur ", comme dans notre chant. Or, il ne suffit pas de se trouver dans de telles dispositions pour arrêter le regard du sain(t) sur soi. Le sain $(t)$ peut également se montrer vraiment terrible et accroître la douleur des demandeurs. Il peut, avant de rétablir la conduite du corps du patient, pousser celui-ci dans ses derniers retranchements, vers la plus extrême dé-possession. C'est pour cela que les hommes ou les femmes ayant subi les plus grandes atteintes de la part de la surnature, les hommes ou les femmes ayant subi la pire des humiliations, sont considérés comme les plus fascinants voire les plus charismatiques. Et c'est là que réside tout l'enjeu de la sphère maraboutique : retourner la plus extrême dé-possession en la plus lumineuse des possessions. Être, à l'instar d'Abd El Kader, maître de son sujet.

Le mauvais œil qui s'ancre dans le réel

Selon David Le Breton, pour ne pas être frappé par l'œil, « Il ne faut jamais donner prise à l'appréciation de l'autre, rester dans la discrétion $»^{21}$. Autant dire tout ce qui est impossible pour nos informateurs. Le marabout, par essence, est fortement assujetti à ce trouble puisqu'il doit accréditer son pouvoir par de nombreux charismes (actes extraordinaires tels que les soins, les divinations etc.) :

Ethn _ Pensez-vous Jamila que la maladie que vous avez on pourrait dire que c'est une conséquence du mauvais œil?

Jamila _ Le mauvais oeil peut causer des malaises. Alors ces malaises-là ou bien ils peuvent se transformer en une grave maladie ou bien ils se transforment en une bonne et belle action, la baraka.

Ethn_C'est un peu ça qui a joué chez vous?

Jamila _Oui.

Ethn _ Pensez-vous que ça, ce bon côté des choses, de l'œil, ça vous a apporté la sensibilité ? La sensation comme on dit. Al-'ih [2]

Jamila _ Oui. Oui, j'ai la sensation, j'ai...

Ethn _ Est-ce que vous pouvez m'expliquer comment ça se manifeste?

Jamila _ Il y a toujours quelque chose soit dans nos doigts, soit dans nos pieds qui nous dit qu'il y a quelque chose.

Ethn _ Qu'il y a quelque chose?

Jamila_Oui.

Ethn _ Ce « quelque chose », c'est quoi?

Jamila _ Ce quelque chose c'est une espèce de... une espèce d'anticipation.

Ethn_ D'anticipation?

Jamila _ D'anticipation, c'est à dire...

Ethn _ Cette sensation, cette sensibilité, c'est...

Jamila _ Cette sensibilité c'est comme un coup de fil. C'est comme quelque chose qui me dit: "Ah! voilà quelque chose qui va t'arriver, qui va... malade, un enfant malade, etc.. »

Ethn _ Est-ce qu'on peut prendre cette sensibilité, cette sensation aussi comme le besoin d'aller vers les autres, d'aider les gens? de donner aux autres?

Jamila _ Bien sûr! Cette sensation me pousse à faire du bien. À faire du bien, à guérir les autres, à leur donner du réconfort. Je suis bien contente lorsque les gens viennent et que je les sauve.

Nous avons rencontré sur le terrain d'autres femmes comme Jamila considérées comme dotées de baraka, notamment une octogénaire prénommée Zidiya. Pendant sa jeunesse, Zidiya s'était éprise d'un paysan qui travaillait pour son mari, un homme très fortuné. 
Ce paysan, quand il était sur les lieux de son labeur, à proximité de la propriété de son patron, chantait, chantait d'une telle façon que Zidiya ne pouvait y rester insensible. Sa voix était très mélodieuse. Il l'a amadouée. Zidiya a quitté sa famille, son mari, tous ses biens, pour cet homme. Ensuite, elle s'est repentie. Elle a appris des techniques, est devenue pieuse, le pouvoir lui est venu. Lors d'un entretien dans sa petite maison bâtie par le voisinage pour remplacer sa vieille tente - une cliente la sollicitait. Nous avons noté :

La femme s'est assise aux côtés de Zidiya. Cette femme avait les yeux hagards. Elle venait parce qu'elle pensait être frappée par l'œil. Elle se plaignait de fatigue. Zidiya a pris son foulard qui lui servait de turban, a mesuré les coudées de ce foulard. À chaque coudée, elle plaçait une épingle ou une allumette qui transperçait le foulard, pour ne pas perdre les mesures. Elle mit le foulard dans sa main, apposa cette main sur tout le corps de la patiente en récitant les paroles suivantes: "eh mauvais œil qui est précieux, sors de ce corps. Si tu appartiens aux femmes, sors des vêtements. Si tu appartiens aux hommes, sors des pierres. Si tu es des esclaves, sors de loin. Si tu es des serviteurs, sors des pieds. Les cheveux ne poussent pas dans la paume de la main. Et l'œil ne peut pas s'y fixer. Cinq et jeudi sel et édad ${ }^{22}$ dans l'œil des envieux, des jaloux ». Zidiya compta de un à sept. Elle tourna son bras dont la main pressait encore le foulard sept fois autour de la patiente. Elle déroula le foulard, vérifia les mesures. À chaque fois, les repères avaient reculé d'environ une dizaine de centimètres, ce qui signifiait que les coudées avaient rétréci, que la malade était frappée par l'œil. Zidiya reprit le foulard dans le creux de sa main, l'appliqua à nouveau sur la malade en renouvelant ses gestes et paroles. Les coudées reprirent leur longueur initiale. Elle recommença le même processus, la longueur ne changea plus, la malade était bien guérie. La femme donna à Zidiya un peu d'argent.

Ethn _ [je demandais à Zidiya, avant que cette cliente ne nous interrompe, si elle était frappée par l'œil] Tout à l'heure vous avez dit que vous aviez eu le mauvais œil, que vous étiez une très belle femme, grosse...

Zidiya_Grosse. Oui.

Ethn _ C'est quoi le rapport?

Zidiya _ C'est que lorsque les gens voient une chose très belle, une chose très bonne,

les gens sont envieux.

Ethn_C'est suite à votre beauté...

Zidiya _ Oui.

Ethn _ Que vous êtes tombée malade?

Zidiya _ Non, ce n'est pas suite à la beauté, c'est suite aux paroles dites par les gens qui m'observaient. Alors, quelques fois, en passant, on dit : « Oh cette chose est une chose merveilleuse ! » et comme ça on la touche avec l'œil.

31 Nous avons également rencontré à Bir El Haffey deux autres femmes guérisseuses: Fatma Zina et Aïcha. Ces femmes ne sont pas de la même famille. Fatma Zina est une Akrimi, Aïcha appartient à la famille des Amor qui eux-mêmes descendent de Sidi Khlifa, un grand marabout dont une mosquée de Gafsa perpétue la mémoire. Aïcha ne s'est jamais mariée et n'a jamais eu d'enfants. Quant à Fatma Zina, elle était habituée, lors de son enfance, à visiter un saint qui se nommait Mohamed Essalah ${ }^{23}$, et cela à chaque fois qu'elle était malade. À force de fréquenter cet homme, Fatma Zina gagna un peu de sa sainteté ; un jour Sidi Mohamed Essalah prit sa main, lut dans sa paume et lui 
dit que maintenant elle était capable de soigner les gens. Lors d'une de nos fréquentes entrevues, Fatma Zina nous dit:

Fatma Zina _ J'ai eu deux maris. J'ai décidé de divorcer, du premier et du deuxième. Je ne peux pas vous dire pourquoi. Je suis timide. Nous n'avions pas le même caractère. Lorsque nous portons un enfant, nous sommes nerveuses, les hommes ne comprennent pas pourquoi. Avec le premier, j'ai eu une seule fille. Avec le deuxième, je n'ai pas eu d'enfant. Le premier était le fils de ma tante. J'ai renoncé à tout lors du divorce. Je suis partie les mains vides. Actuellement je n'ai pas de problèmes avec ma tante. Mon cousin, j'essaie de l'éviter. Après cette séparation, les gens ont fait de nombreuses démarches pour que je retourne à ses côtés. Je n'ai pas accepté. Même mes vêtements, j'y ai renoncé. Je suis retournée dans ma famille, ils m'ont pris en charge. Puis j'ai confectionné des couvertures, des draps. Ce divorce n'a pas posé de nombreux problèmes. Le deuxième mari, je n'avais pas l'intention de me marier avec lui. Ce sont mes parents qui ont décidé. Il n'était pas de ma famille. Il travaillait en Libye. Je suis resté avec lui cinq ou six ans. Mais en réalité il était toujours en déplacement. Notre vie conjugale a duré au maximum une année. Mais il avait mauvaise réputation, il était alcoolique. Moi je voulais être seule. Les mariages m'étaient imposés. Seule, pour être libre, ne pas avoir de problèmes, me consacrer qu'à la prière. Avoir une vie spirituelle.

Ethn _ Aïcha et vous avez eu une vie différente. Vous avez eu deux maris, elle est restée célibataire. Qu'avez-vous gagné l'une de l'autre?

Fatma Zina _ Nous sommes des sœurs, la providence nous a unies. Nous avons des techniques différentes, chacune sa tarik ${ }_{2}^{[} a^{24}$. Aïcha est là depuis trente-cinq ans. Elle vivait au début avec son défunt frère Lazar. Dès notre première rencontre, elle a eu de l'affection pour moi. Le désir que nous nous unissions dans la vie. Elle est venue dans ma maison paternelle. Notre famille était heureuse. Sa famille également. Ils voyaient qu'elle était dans de bonnes mains.

Avec Fatma Zina, la figure de la femme phallique "apparaît». Ces lignes, mais également les propos de nos autres informatrices, rapportées au portrait d'Aïcha Qondicha dépeint par Bertrand Hell, ne diffèrent guère et possèdent en eux tout le poids du signifiant œil. Bertrand Hell écrit : « [la] féminité [d'Aïcha Qondicha] se place sous le signe de la mélancolie, de la lascivité et de l'irascibilité [...] Aicha la fière, la solitaire, incarne la liberté, l'insoumission face à la toute puissance masculine. $»^{25}$ L'intervention d'Aïcha Qondicha et la menace qu'elle fait peser sur les généalogies (la progéniture de Sidi Abd El Kader Djilani), semble nous indiquer que toutes ces figures de djinn, qu'elles soient féminines ou masculines (les ancêtres), n'ont d'autre objet que de permettre à la communauté par le biais du marabout, de pénétrer dans le territoire de la personne menaçante risquant de porter le danger sur son groupe. Comme si le mal - la transgression - n'était autre qu'un virus qui pourrait contaminer l'ensemble des coreligionnaires de la personne déviante et stigmatisée. En ce sens, notre analyse ne s'éloigne pas de celle des auteurs qui perçoivent la baraka comme un fluide pouvant imprégner tous ceux qui «s'imbibent» de la personne élue. Or, cette personne élue, avant de subir le schéma de l'initiation, pouvait également les imprégner, mais d'une toute autre manière : elle pouvait les contaminer. La baraka était au stade de mauvais objet. Il fallait la dompter.

L'élection agit comme le moment où se retourne l'identité. Le moment où tous les rôles, tous les visages que revêt l'individu, peuvent être changés, modifiés, remis en question; le moment le plus carnavalesque qu'il soit. Celui où tout est permis. Pour s'en convaincre, il suffit de s'en remettre aux signifiants de l'œil. Fatma Zina soigne également le mauvais œil : elle utilise la technique du foulard, comme Zidiya, ainsi 
qu'une autre technique qui se nomme al-shab. Il s'agit de prendre un morceau de pierre que l'on place dans un brasero. Si le patient est une femme et qu'elle est frappée par le mauvais œil, le morceau de pierre prendra l'apparence du sexe de la femme, s'il s'agit d'un homme, il prendra l'apparence du sexe masculin. Le thérapeute tient comme un lien de cause à effet le trouble de l'œil et l'identité sexuelle de la victime. Rien qu'au niveau des techniques, ces ambiguïtés sont belles et bien présentes. Or, tirer de telles constatations revient à mettre en branle une partie de l'édifice baraka qui est véhiculé dans le sens commun de la région observée. Cependant, de telles constations prennent place dans le schéma que nous avons dressé et avec certains propos d'autres auteurs. Crapanzano aurait également relevé ce type d'ambiguïtés auprès des Hamadcha du Maroc. En analysant les légendes portant sur des personnages éponymes de ses lieux d'investigations, Crapanzano constate: «la légende porte sur le problème de l'identité masculine. Elle décrit symboliquement certains conflits sexuels [...] une partie au moins des Hamadcha souffrent de problèmes d'identité masculine [...] $»^{26}$.

Concernant toujours le Maroc, Bertrand Hell écrit : "La maladie ne se définit pas à l'aune des dysfonctionnements corporels qu'elle provoque. Elle est interprétée chez les Gnawa comme une marque de l'invisible, le signe possible d'une élection. À ce titre la maladie joue un rôle central dans le parcours individuel des affiliés : véritable passage obligé pour les grands initiés, elle apparaît comme le point de départ du cheminement initiatique. La maladie se définit comme une cassure qui permet d'ouvrir les yeux $»^{27}$. Les troubles de l'identité semblent être tout aussi déterminants dans le parcours initiatique des affiliés Gnawa et Hamadcha que dans celui de nos initiés de Bir El Haffey. $\mathrm{Au}$ Maroc, ils sont synonymes de déboires liés à des questions de morale et de transgressions, qui ne sont toutefois pas très banales quand on observe de près ce qui se joue au sein des confréries mentionnées. On s'y autorise des conduites et des attitudes qui sont loin d'être admises dans les autres secteurs de la société marocaine. Bertrand Hell parlera d'exutoire. Mais ce type de rituel semble être assez extrême et ne peut être une vraie représentation des conduites préconisées dans les rituels magicoreligieux, qu'il s'agisse des autres courants de la société marocaine ou même tout simplement des figures tunisiennes que nous avons été amenées à rencontrer. Cependant, il semble également que, dans l'imaginaire tunisien, les formes de transgressions extrêmes peuvent être synonymes, parallèlement, d'une très grande maitrise de la surnature, et plus généralement de la baraka. Car il ne fait pas de doute que si les attitudes de certains rituels marocains étaient permises, beaucoup de personnes s'y adonneraient. Cependant, à Bir El Haffey, l'environnement est très rigoriste voire même puritain et les excès du maraboutisme marocain apparaissent aux personnes que nous avons questionnées à ce sujet comme une pure folie. À Bir El Haffey, les habitants transgressent, mais pas trop. De même, ils pratiquent, mais pas trop non plus.

De tels constats peuvent sembler être des lieux communs de la discipline ethnologique sans que pour cela, un objet tel que l'œil ait été mobilisé. Cependant la thèse des génies comme alliés a été également mise en évidence par de nombreux autres auteurs: Germaine Tillion écrit : "Dans les régions où se propagea massivement l'endogamie c'est à dire les rivages méditerranéens - , le "mariage en famille"a pu, ça et là, apparaître parfois oppressant à ceux et à celles auxquels il était imposé. Surgissent alors des djinns et diablotins "consolateurs-persécuteurs" des gamins incompris et des épouses mal mariées : les maumariées de nos complaintes... (ce serait, en tout cas, une des façons d'expliquer leur présence dans la zone la plus anciennement civilisée du 
monde). $\aleph^{28}$ D'autres auteurs peuvent nous renseigner à ce sujet bien que leur objet de recherche semble, à première vue, très éloigné de nos préoccupations.

Il n'est pas anodin qu'une partie de cette étude soit consacrée au phénomène de la prostitution puis une autre au phénomène des femmes pratiquant le maraboutisme, ces deux groupes de femmes apparemment distincts ont la particularité de s'être immiscés dans des espaces qui leur étaient interdits. Aussi, dans une étude portant sur les femmes marginales en terre d'Islam, Dalenda et Abdelhamid Largueche écrivent: "Emile Dermenghem nous a minutieusement décrit les formes de prostitution des femmes Neilia et des 'Azriat d'Algérie comme fait de culture et non de crise. Il s'agissait de formes de prostitution et de pratiques assumées et intégrées dans les valeurs de la tribu qui permettait à ses filles de se prostituer un certain nombre d'années en errant à travers les cités d'Algérie et du Maghreb, pour réintégrer ensuite leurs villages et se marier [...] Nous savons par ailleurs qu'en Tunisie, pays plus homogène humainement que l'Algérie, de telles singularités constituaient plutôt un fait rare. Mais certaines fractions de tribus du centre ou du nord-ouest étaient connues par certaines fonctions particulières et propres aux femmes qui parcouraient à la saison chaude les villes et villages pour "lire le destin" des hommes. C'étaient les célèbres deggaza (voyantes ou liseuses de destin). La tradition orale et vécue, jusqu'à une date récente, présentait ces filles comme ayant un comportement sexuel assez libéral, ne voyant souvent pas d'inconvénient à charmer et à séduire le premier homme qui leur plaisait. $»^{29}$ Nous avons appris auprès de nos informateurs que ces femmes ne relèvent pas que de la légende. De plus, leurs particularités et l'origine de leurs pouvoirs proviennent d'une élection très similaire à celle des femmes évoquées dans notre étude. Tout se passerait donc comme si la maîtrise de l'invisible pouvait constituer un vecteur d'éloignement vis-à-vis d'une certaine pesanteur sociale. Cependant, le maraboutisme tunisien n'est pas le maraboutisme d'autres terres d'Islam. Le cas de la deggaza serait une forme paroxystique de cette émancipation. Car les familles de Bir El Haffey accordent un peu plus de liberté à une femme initiée mais cette émancipation va toujours de paire avec certains sacrifices. Les deux partis trouvent des compromis.

Conclusion

37 Si l'initiation permet aux femmes d'accéder à des activités qui leur sont normalement interdites, c'est que cette activité les virilise. Parallèlement, les hommes sous-estiment l'œil parce que, dès la naissance, ils sont destinés à l'espace public. Évoluer sous les yeux des autres est le rôle qu'on attend d'eux. Or, si l'homme est atteint par l'œil - ce que la technique nommée al-shab de Fatma Zina et celle du foulard de Zidiya nous prouvent - c'est que le rôle qu'on attend de lui n'a pas été réalisé. Les canons de la masculinité ne sont pas respectés. Seulement, la littérature ethnologique, ainsi que bon nombre de mythes, nous laissent à penser qu'une fois l'initiation achevée, la figure de la puissance est celle qui sert à caractériser tous les marabouts. Or, le maraboutisme ne serait qu'une illusion de la puissance. Il permettrait seulement de s'accommoder avec ce que l'on est. L'homme et la femme subissant une initiation ne chercheraient pas le pouvoir, la richesse, la reconnaissance de leur charisme, mais tenteraient seulement et modestement d'imposer les ambiguïtés de leurs personnalités.

Depuis son élection, les proches de Jamila précisent qu'elle soigne les malades. Elle est devenue humble, modeste, endurante et pieuse. De plus, elle soigne avec sa main gauche, le côté féminin par excellence ${ }^{30}$. Or, tout ceci a été obtenu après le truchement de l'initiation. Car, avant son élection, Jamila était tout le contraire. Elle était en proie à 
la peur, la solitude et le mauvais oeil. Ce qui suscita l'attaque des génies qui n'étaient en fait que des attaques de son environnement familial proche. L'informatrice, à ce stade de son histoire, savait sûrement que son comportement n'était pas tolérable et il lui fallait trouver une porte de sortie. Nous avons observé que cette porte de sortie fut le maraboutisme. Ce changement fut possible parce que l'existence que Jamila décida volontairement, ou sous le joug des génies, d'enterrer, lui permit de développer la condition essentielle, celle de la sensibilité décrite comme al-'ih [5] du mauvais œil. De plus, cette évolution fut concrétisée par un changement tout aussi remarquable et voulu par elle seule. Jamila choisit de changer de nom lorsqu'elle franchit le cap de l'élection: avant d'exercer, elle se prénommait, comme nous le faisons depuis le début de cette étude, Jamila, nom qui lui avait été donné par sa famille à sa naissance. Mais, depuis qu'elle exerce (environ neuf années à ce jour), elle ne se fait plus appeler par ce nom qui signifie en langue arabe la «beauté ». Elle a choisi de se «rebaptiser » Neiijima, ce qui veut dire : la « capacité», le " pouvoir ». Probablement l'origine, nous l'avons vu, de ses troubles et, en particulier, l'origine de l'œil, puisque rien ne peut attirer l'œil plus que la beauté. Rien, si ce n'est la puissance d'un homme.

\section{BIBLIOGRAPHIE}

AMRI Nelly \& Laroussi, Les femmes soufies ou la passion de Dieu, Dangles, 1992.

BOURDIEU Pierre, Le sens pratique, Paris, Les éditions de minuit, 1980.

DUVIGNEAU Jean, Chébika suivi de Retour à Chébika 1990, Changements dans un village du Sud Tunisien, Paris, Plon, 1991.

CRAPANZANO Vincent, Les Hamadcha, Une étude d'ethnopsychiatrie marocaine, Paris, Sanofi-Syntélabo, 2000.

EL BACHARI Mohammed, Homme dominant, homme dominé : l'imaginaire incestueux au Maghreb, Paris, L'Harmattan, 1999.

HELL Bertrand, Le tourbillon des génies, Paris, Flammarion, 2002.

LARGUECHE Dalenda \&Abdelhamid, Marginales en terre d'Islam, Tunis, Cérès, 1992.

LE BRETON David, Les passions ordinaires. Anthropologie des émotions, Paris, Armand Colin/ Masson, 1998.

OUITIS Aïssa, Possession, magie et prophétie en Algérie, Paris, Arcantère, 1984.

TILLION Germaine, Il était une fois l'ethnographie, Paris, éditions du Seuil, 2000.

\section{NOTES}

1. Faute de ne disposer d'un terme qui fasse l'unanimité au sein de la discipline, nous emploierons le terme de « maraboutisme » tout le long de cette étude. 
2. La seule étude qui leur est entièrement consacrée est celle de Nelly \& Laroussi Amri (AMRI, 1992), étude qui a pour principal cadre le courant soufi.

3. Bir El Haffey appartient au gouvernorat de Sidi Bouzid, création récente de l'état tunisien. Bir El Haffey se situe dans une région comprenant la ville de Sidi Bouzid au nord-est, la ville de Gafsa au sud et les villes de Sbeïtla et Kasserine plus à l'ouest.

4. En arabe dialectal de la région :'arrāf pour un homme. Toutes les translittérations des termes arabes de cette étude seront effectuées selon les nomenclatures de

l'encyclopédie de l'Islam : Encyclopédie de l'Islam, Leiden, Brill, 1961-2005. Tous les termes arabes employés par les auteurs cités seront retranscrits tel que dans le texte. Quant aux noms propres, ils seront écrits à la manière courante en Tunisie.

5. En arabe dialectale de la région : mabrūk pour un homme.

6. DUVIGNAUD, 1991, p. 142.

7. OUITIS, 1984, p. 78.

8. Le terme marabout sert aussi bien à désigner la personne douée de baraka que le lieu où elle repose.

9. Il est intéressant de préciser que le prénom de cette femme signifie « la mère de la prospérité ». Or nous observerons par la suite que cette prospérité qui est censé caractériser sa progéniture peut être une des causes du trouble de son fils. Car Om El Khir a également donné naissance à deux autres enfants de sexe masculin, ce qui, pour les populations de ces régions, est considéré comme une bénédiction mais peut également susciter beaucoup de jalousie et d'envie au sein de son environnement. C'est de cette dernière attitude qu'est censé provenir le mauvais œil.

10. Les génies sont censés détester l'odeur que dégage cette graine.

11. Notre terrain dura entre avril 2002 et avril 2004 et fut entrecoupé par deux brefs retours vers la France.

12. Certaines informations rencontrées sur le terrain viennent contredire cette dernière observation.

13. HELL, 2002, p. 97.

14. Le bindìr est de forme cylindrique et est recouvert par une peau de chèvre tendue régulièrement au-dessus d'un feu. L'autre percussion très répandue dans la région est la darbūka. La darbūka, contrairement au bindīr, est plutôt utilisée dans des cérémonies profanes et est parfois accompagnée par le mizwid, cornemuse locale.

15. Ibid. p. 336.

16. Nous pouvons notamment citer Vincent Crapanzano (CRAPANZANO, 2000) et Mohammed El Bachari (EL BACHARI, 1999).

17. Ibid. p. 339-340.

18. Abd El Kader est surnommé en Tunisie Djallūl, le «boiteux », celui qui endure les épreuves avec dignité.

19. Ibid., p. 130.

20. Ibid., p. 130.

21. LE BRETON, 1998, p. 193.

22. Une plante de montagne. Nous nous excusons auprès du lecteur de n'avoir pu retranscrire ce terme correctement.

23. Sidi Mohamed Essalah est un magérien, un membre d'une famille nommée Mager. Ce personnage est aussi de parenté avec un grand saint libyen qui se nomme Sidi Abdessalem Lassmer, un descendant du prophète Mohamed et de sa fille Fatima. 
24. Dans les traditions musulmanes, ce terme représente les voies ou confréries soufies. Mais, dans la région de Bir El Haffey, ce terme sert à désigner les techniques des guérisseurs.

25. Ibid. p. 340-341.

26. CRAPANZANO, 2000, p. 198.

27. HELL, 2002, p. 54.

28. TILLION, 2000, p. 97.

29. LARGUECHE, 1992, p. 36.

30. BOURDIEU, 1980 , p. 354.

\section{RÉSUMÉS}

Au Maghreb subsiste un Islam populaire organisé autour de la baraka, don ou grâce possédé par les «marabouts», personnages qui peuvent, entre autres, accomplir des guérisons et servir de médiateurs entre le monde ici-bas et l'au-delà. À Bir El Haffey, petite ville du centre tunisien où furent menées nos observations, les femmes représentent un maillon non-négligeable de cette pratique d'autant qu'elles ont la particularité de s'être immiscées dans l'espace public, traditionnellement réservé aux hommes. Cette appropriation, perçue comme une conduite marginale et transgressive, fait de ces femmes des victimes du mauvais œil, attaque magique du regard provoquant un trouble de l'identité. L'objectif de cette étude est de démontrer que ce trouble, ancré dans la vie des pratiquantes du maraboutisme, fait non seulement écho aux grandes figures mythiques qui peuplent les monographies de cette aire culturelle mais leur permet également de développer les qualités essentielles de leur vocation tout en légitimant certaines ambiguïtés de leurs personnalités.

In northern Africa remains a popular Islam revolving around the notion of baraka, a kind of gift or grace shared by the marabouts that allows them to accomplish healing acts and be a connector between earth and the beyond. In Bir El Haffey, a town located in central Tunisia, women do play a specific role in this practice as part of the public landscape, usually exclusively devoted to men. But such a transgression, a marginal behaviour present these women as victims of the evil eye, a magical attack of the sight bringing identity disorder. The objective of this study is to show that this disorder, deeply related to the life of these women practising maraboutism is not only linked to the great mythical figures found in the monographs of this culture but it also allows these women to develop essential qualities of their vocation while legitimating some ambiguities of their personalities.

\section{AUTEUR}

\section{SALEM AKRIMI}

Ethnologue, spécialisé en anthropologie religieuse, auteur d'une thèse de doctorat à l'Université Paul Verlaine de Metz intitulée « Une anthropologie comparative du don et de la baraka ; quelques exemples sur le maraboutisme tunisien ». 